\title{
Review: the Prentif cervical cap was as effective as the diaphragm in preventing pregnancy, and the FemCap was not
}

\author{
Gallo MF, Grimes DA, Schulz KF Cervical cap versus diaphragm for contraception. Cochrane Database Syst Rev \\ 2002;(4):CD003551 (latest version 22 Aug 2002).
}

\section{QUESTION: Is the cervical cap more effective, safe, and acceptable than the diaphragm for contraception?}

\section{Data sources}

Studies were identified by searching Medline, Popline, Cochrane Controlled Trials Register, EMBASE/ Excerpta Medica, and LILACS; reviewing the references of pertinent articles; and contacting manufacturers and investigators in the field.

\section{Study selection}

Studies were selected if they were randomised controlled trials in any language comparing a cervical cap with a diaphragm in women of reproductive age with no contraindications to either barrier method.

\section{Data extraction}

2 reviewers independently extracted data on study quality (design, blinding, randomisation method, allocation concealment, follow up, and discontinuation rates), participant characteristics, type of cervical cap and diaphragm, and results. Extracted data were verified by a second reviewer. Outcomes were contraceptive efficacy, discontinuation, safety, and acceptability.

\section{Main results}

2 trials met the selection criteria: 1 trial compared the Prentif Cavity Rim Cervical Cap (Lamberts Ltd, Oxford, UK) with the Ortho diaphragm (Ortho Pharmaceutical Corporation, Raritan, NJ, USA) ( $\mathrm{n}=1529,6-24$ mo follow up), and 1 trial compared the FemCap cervical cap (rubber manufactured by Hi-Tech Rubber, Anaheim, CA, USA) with the All-Flex diaphragm (Ortho) ( $\mathrm{n}=841,28 \mathrm{wk}$ follow up). The Prentif cap was as effective as the diaphragm in preventing pregnancy (24 mo follow up); the FemCap was not as effective as the diaphragm (6 mo follow up) (table). Papanicolaou smears showed greater risk of Class I to Class III cytological conversion with the Prentif cap than the diaphragm (odds ratio [OR] 2.31, 95\% CI 1.04 to 5.11); rates of abnormal Papanicolaou smears did not differ between FemCap and diaphragm users. Prentif cap users had lower risk of vaginal ulcerations or lacerations than diaphragm users (OR 0.31, CI 0.14 to 0.71 ). More FemCap users reported blood on the device on removal than diaphragm users (OR 2.29, CI 1.27 to 4.14). Dislodgement of the device was reported by more users of both the Prentif cap (OR 4.10, CI 3.18 to 5.30) and the FemCap (OR 5.46, CI 3.74 to 7.96) than users of the diaphragm. Discontinuation rates did not differ between Prentif cap and diaphragm users or between FemCap and diaphragm users.

\section{COMMENTARY} using a cervical cap. transmission. $^{2}$

\section{Conclusions}

The Prentif Cavity Rim Cervical Cap was as effective as the diaphragm in preventing pregnancy; the FemCap cervical cap was not as effective as the diaphragm. More cytological changes in the cervix occurred with the Prentif cap than the diaphragm; the FemCap did not differ from the diaphragm for cytological changes.
Sources of funding: National Institute of Child Health and Human Development and US Agency for International Development.

For correspondence: Ms $M$ F Gallo, Family Health International, Research Triangle Park, NC, USA. mgallo@fhi.org

Although several cervical caps are marketed, Gallo et al identified just 2 randomised controlled trials comparing any type of cervical cap with diaphragms. This was despite a rigorous and extensive strategy to locate all relevant studies. Any review is limited by the quality of the studies it includes. The review by Gallo et al included only 2 trials, which were limited because they either did not use methods known to reduce systematic bias in analyses (eg, intention to treat analysis) or did not adequately describe the methods to reduce such bias. Although it is lamentable that such an important medical device can be marketed in the absence of high quality population based studies, this is the best available evidence.

The results of this review are relevant to public health nurses, nurse practitioners, clinical nurse specialists, and nurses who work in gynaecological offices, birth control clinics, and family planning centres. Although female barrier contraceptives are not widely used, ${ }^{1}$ the findings provide useful information about pregnancy risk and adverse effects with cervical caps and diaphragms. Specifically, when choosing between the cap and the diaphragm, women need to know that the Prentif cap, unlike the FemCap, is as effective as the diaphragm for preventing pregnancy. However, it is important to note that the overall risk of pregnancy with either contraceptive method over the trial periods was between 1 in 8 to 1 in 20 women using a diaphragm, and 1 in 6 to 1 in 8 women

Overall, the diaphragm probably remains the female barrier method of choice among the non-hormonal alternatives. It may have a lower pregnancy rate, low risk of Class I to Class III cytological conversions, low dislodgment rate, and fits easily. The cervical caps either did not differ from the diaphragm for these outcomes, or were less effective. However, women need to consider their individual risk of HIV or sexually transmitted disease when choosing a contraceptive method because the diaphragm and cervical caps offer little protection, and spermicide (nonoxynol-9) may increase the risk of HIV

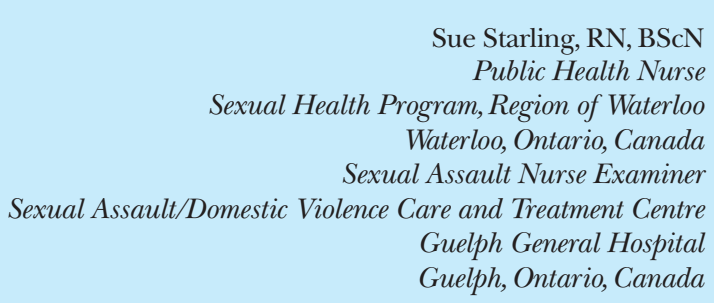

Guelph, Ontario, Canada

1 Piccinino LJ, Mosher WD. Trends in contraceptive use in the United States: 1982-1995. Fam Plann Perspect 1998;30:4-10, 46.

Health Canada, Centre for Infectious Disease Prevention and Control. HIV/AIDS Epi Update. Nonoxynol-9 and the risk of HIV transmission. April 2002. http://www.hc-sc.gc.ca/pphb-dgspsp/publicat/epiu-aepi/hiv-vih/nonoxynol_e.html

Cervical cap $v$ diaphragm for contraception *

\begin{tabular}{lllllll} 
Outcome & Number of trials & Follow up & Comparison & Event rates & RRI (95\% Cl) \\
Pregnancy & 1 & 24 months & Prentif cap $v$ diaphragm & $15.4 \% v 12.4 \%$ & $21 \%(-10$ to 61$)$ \\
\hline & 1 & 6 months & FemCap $v$ diaphragm & $9.5 \% v 5.5 \%$ & $71 \%(1$ to 187$)$ & $25(13$ to 660$)$ \\
\hline
\end{tabular}

*Abbreviations defined in glossary; RRI, NNH, and $\mathrm{Cl}$ calculated from data in article using a fixed effects model. 